\title{
Wakefield Generation in a Plasma Filled Rectangular Waveguide
}

\author{
Anil Kumar Aria and Hitendra Kumar Malik*
}

Plasma Waves and Particle Acceleration Laboratory, Department of Physics, Indian Institute of Technology Delhi, New Delhi-110 016, India

\begin{abstract}
We study the wakefield in a plasma filled rectangular waveguide based on a differential equation derived in terms of the wake potential $\phi$, when the pulse duration $\tau$ matches with inverse of the plasma frequency $f_{p}^{-1}$. This equation is solved using fourth-order Runge-Kutta method for the Gaussian-like profile of the microwave pulse. The effects of microwave frequency $f$, waveguide width $b$ and microwave intensity $I$ are investigated on the wakefield $E_{W}$. The amplitude of the wakefield is found to increase with microwave pulse duration and its intensity, but it gets decreased with microwave frequency and waveguide width. By optimizing various parameters, we can achieve wakefield of appreciable strength for the purpose of particle acceleration.
\end{abstract}

Keywords: Microwave pulse, plasma, pulse duration, rectangular waveguide, wakefield, wake potential.

\section{INTRODUCTION}

The subject charged particle acceleration has received worldwide attention due to its varied applications in the field of nuclear physics, high energy physics, harmonic generation, free electron lasers, material science, etc. Plasma based accelerators stand first because of their ability to sustain extremely large acceleration gradients, which are approximately three orders of magnitude greater than that achieved in conventional RF linacs. The use of laser plasma accelerators has been made in radio isotope production through $(\gamma, n)$ reactions with laser accelerated electron bunches in the range tens of $\mathrm{MeV}[1,2]$. The short pulse nature and high charge of the accelerated bunches also has applications in the production of coherent terahertz $(\mathrm{THz})$ radiation, which is achieved when femtosecond electron bunches cross the plasma vacuum boundary and emit transition radiation [3-5]. Another application is in the generation of femtosecond $\mathrm{X}$ ray pulses produced by the betatron radiation emitted when the electron beam propagates through the plasma.

Plasma based acceleration schemes mainly include the plasma beat wave accelerator, laser wakefield accelerator, $\vec{v}_{p} \times \vec{B}$ accelerator, plasma wakefield accelerator, etc. Wakefield generation for the purpose of particle acceleration has been widely studied experimentally, analytically and using simulations [6-20]. For the purpose of efficient acceleration, it is necessary to excite the wakefield of a large amplitude along with its speed nearly equal to the speed of light. The wakefield is reported to be enhanced by the nonlinearities in response of plasma to ponderomotive force of a long smooth laser pulse of relativistic intensity whose pulse

*Address correspondence to this author at the Plasma Waves and Particle Acceleration Laboratory, Department of Physics, Indian Institute of Technology Delhi, New Delhi-110 016, India;

Tel: 91-11-2659-1303; Fax: 91-11-2658-2037/2658-2277;

E-mail: hkmalik@physics.iitd.ac.in, hkmalik@hotmail.com length is much larger than the half of the plasma wavelength [21]. The amplitude of the laser wakefield has also been found to increase by the ionization processes of the gases at comparatively higher laser peak intensities [22]. A capillary tube can be used as a waveguide in order to enhance the interaction length [23]. Tapered plasma channels have been proposed for the enhancement of interaction length to achieve greater acceleration [24]. On the other hand, efforts have been made related to wakefield excitation by relativistic electron bunch [19, 20], and coupling of longitudinal and transverse motion of accelerated electrons in laser wakefield [8]. Lotov [7] has analytically studied the laser wakefield acceleration in narrow plasma filled channels. With regard to the importance of polarization effects, Kado et al. [25] have observed strongly collimated proton beam from Tantalum targets when irradiated with circularly polarized laser pulses. With the help of radially polarized ultra relativistic laser pulses, Karmakar and Pukhov [26] have shown that collimated attosecond $\mathrm{GeV}$ electron bunches can be produced by ionization of high- $\mathrm{Z}$ material. They also compared the results with the case of Gaussian laser pulses and found that the radially polarized laser pulses are superior both in the maximum energy gain and in the quality of the produced electron beams. Xu et al. [27] made a comparison between circularly polarized (CP) and linearly polarized (LP) fields with regard to the laser driven electron acceleration in vacuum and found that the CP field can give rise to greater acceleration efficiency.

Analytical investigations on wakefield acceleration using a dielectric lined waveguide structure showed the acceleration gradient for electrons or positrons in the range of 50-100 $\mathrm{MV} / \mathrm{m}$ for a few $\mathrm{nC}$ driving bunches [28]. In another wakefield accelerator, a peak acceleration gradient of $155 \mathrm{MeV} / \mathrm{m}$ was predicted for a $2 \mathrm{nC}$ rectangular drive bunch [29]. Jing et al. [30] have found transverse wakefield of about 0.13 $\mathrm{MeV} / \mathrm{mnC}(0.2 \mathrm{MeV} / \mathrm{mnC}$ ) due to $\mathrm{X}$-dipole modes (Y-dipole modes) in an $\mathrm{X}$-band structure generated by an electron 
bunch in dipole-mode wakefield in a waveguide accelerating structure. On the other hand, microwaves have been used in experiments for the particle acceleration via $\vec{v}_{p} \times \vec{B}$ process [31-33] and resonance absorption during wave particle interaction [34]. Short microwave pulses have also been used in some experiments to excite a nonlinear large amplitude ion wave at resonance absorption region [35]. In different type of approach, Jawla et al. [36] have shown that the modified mode excited in a plasma filled waveguide under the effect of an external magnetic field can be quite useful for the electron acceleration using high intensity microwaves. Kumar and Malik [37] have discussed the importance of obliquely applied magnetic field to an electron acceleration and obtained that the larger acceleration is possible when the condition $\omega_{p}>\omega_{c}\left(\omega_{p}\right.$ is the electron plasma frequency and $\omega_{c}$ is the electron cyclotron frequency) is achieved in the plasma filled waveguide. Also, recently it was proposed to use the field of superposed mode in waveguide for the effective electron acceleration [38].

This is clear that except some attempts in waveguides for the wakefield excitation by electron bunches [28-30] the lasers have been extensively used for the purpose of particle acceleration [6-24], and noticeable amount of energy gain has been achieved for the electrons and protons. These types of systems cost very high and very sophisticated instrumentation is also required for the specific/accurate measurement on ultra fast time scale. However, in view of low cost structure and significant acceleration by moderate intensity microwaves, we investigate here the wakefield excited by Gaussian-like microwave pulse in a rectangular waveguide filled with homogeneous plasma. The present calculations and the results would be beneficial for the development of particle accelerator using microwaves in place of ultrahigh lasers.

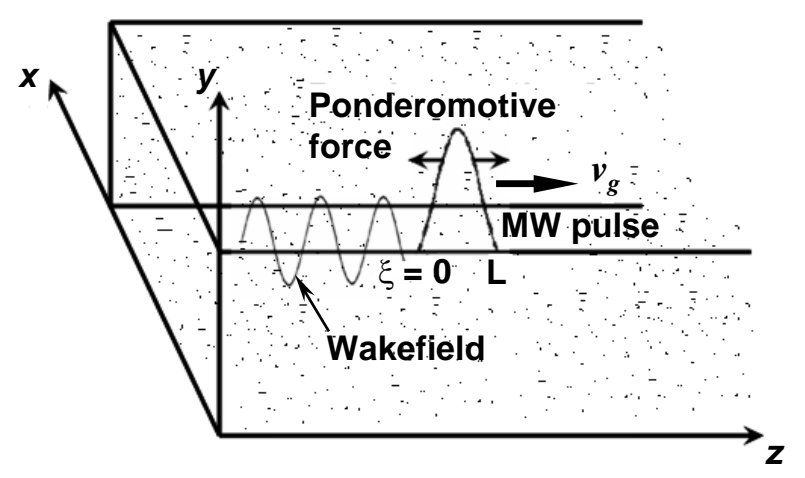

Fig. (1). Schematic of wakefield generation in plasma filled rectangular waveguide by microwave pulse. Here $\xi=z-v_{g} t$ and $L$ is the pulse width.

\section{BASIC FORMULATION}

We consider that a microwave pulse of pulse duration $\tau$ at a frequency $f$ propagates in a plasma filled rectangular waveguide of dimensions $b \times h \mathrm{~m}^{2}$ whose width is $b$ along $\mathrm{x}$-axis and the height $h$ along $\mathrm{y}$-axis. This pulse reso- nantly excites the wakefield (corresponding potential $\phi$ ) in the waveguide under the action of ponderomotive force (Fig. 1) when the pulse duration $\tau$ matches with the inverse of the plasma frequency $f_{p}$, i.e. $\tau=f_{p}^{-1}$. The plasma with ambient density $n_{0}$ is assumed to be cold and collisionless, and the motion of ions is neglected in view of high frequency microwave field. We take $n_{e}$ and $n_{i}$ as the densities of the electrons and ions, respectively, The electric and magnetic fields associated with the microwave are represented by $\vec{E}$ and $\vec{B}$. We use fluid approach and write below the basic fluid equations together with the Maxwell's equations describing the propagation of microwave pulse in the plasma.

$\frac{\partial n_{e}}{\partial t}+\vec{\nabla} \cdot\left(n_{e} \vec{v}\right)=0$

$\frac{d \vec{p}}{d t}+n_{e} e(\vec{E}+\vec{v} \times \vec{B})=0$,

$\vec{\nabla} \cdot \vec{B}=0$,

$\vec{\nabla} \times \vec{B}-\frac{1}{c^{2}} \frac{\partial \vec{E}}{\partial t}-\mu_{0} \vec{J}=0$,

$\vec{\nabla} \times \vec{E}+\frac{\partial \vec{B}}{\partial t}=0$,

$\varepsilon_{0} \vec{\nabla} \cdot \vec{E}_{W}-e\left(n_{i}-n_{e}\right)=0$.

In the above equations, $\vec{J}=-n_{e} e \vec{v}$ and the electrostatic field $E_{W}=-\frac{\partial \phi}{\partial z}$ is along z-axis together with $\phi$ as the wake potential. We assume that the microwave pulse propagates in homogeneous plasma without change in its shape and its electric and magnetic field components in the waveguide made of perfectly conducting material are given by

$$
\begin{aligned}
& \vec{E}=\hat{y} E(\xi) \sin \left(\frac{\pi x}{b}\right), \text { and } \\
& \vec{B}=\hat{x} B_{x}(\xi) \sin \left(\frac{\pi x}{b}\right)+\hat{z} B_{z}(\xi) \cos \left(\frac{\pi x}{b}\right) .
\end{aligned}
$$

The ponderomotive force of microwave pulse expels the electrons from their equilibrium position. We consider the perturbation in electron density as $n_{e 1}$ and take $n_{e}=n_{0}+n_{e 1}$, whereas the ion density $n_{i}=n_{0}$ in view of their heavy mass. With the help of this we linearize the above equations. The linear system is further simplified by transforming to a frame comoving with the microwave pulse and assuming the system to be nonevolving, i.e. all the quantities depend only upon $\xi=z-v_{g} t$, where $v_{g}$ is the group velocity $[15,18,24,39]$. This is done in order to analyze the wakefield in the frame of reference which is stationary with respect to the wake plasma wave, as this plasma wave is excited by the ponderomotive force created by the photons with the phase velocity equal to the group velocity $v_{g}$ of the 
pulse [39]. In some studies this transformation is carried out at the level of phase velocity $c$ of the laser in underdense plasmas as the group velocity $v_{g} \approx c[12,13,22,40]$. As per the mechanism for generating the wake under the effect of given field of microwave the electrons set transverse oscillations in y-direction and pick up the momentum from the pulse along z-direction [39]. Under this situation, the velocity component $v_{z}$ remains dominating and without loss of generality we can neglect the variation of component $v_{x}$ along the waveguide width, i.e. $\partial v_{x} / \partial x=0$. Further in view of compression and rarefaction of electrons along z-axis a pressure gradient (density gradient) develops in z-axis, so the variation of density along $\mathrm{x}$-axis can be neglected, i.e. $\partial n_{e 1} / \partial x=0$. We decompose Eqs. (1)-(6) into their components by taking $\vec{\nabla}$ in (x-z) plane. Under these conditions, we obtain the following equation from Eqs. (1)-(6) for the homogenous plasma where $\partial n_{0} / \partial \xi=0$.

$$
\begin{aligned}
& -v_{g} \frac{\partial n_{e 1}}{\partial \xi}+n_{0} \frac{\partial v_{z}}{\partial \xi}+\frac{\partial n_{e 1} v_{z}}{\partial \xi}=0, \\
& -v_{g} \frac{\partial v_{x}}{\partial \xi}+v_{z} \frac{\partial v_{x}}{\partial \xi}+\frac{e}{m} v_{y} B_{z 0}(\xi) \cos \left(\frac{\pi x}{b}\right)=0, \\
& -v_{g} \frac{\partial v_{y}}{\partial \xi}+v_{x} \frac{\partial v_{y}}{\partial x}+v_{z} \frac{\partial v_{y}}{\partial \xi}+\frac{e}{m}\left(\begin{array}{l}
(\xi) \sin \left(\frac{\pi x}{b}\right)-v_{x} B_{z 0} \cos \left(\frac{\pi x}{b}\right)+ \\
v_{z} B_{x 0}(\xi) \sin \left(\frac{\pi x}{b}\right)
\end{array}\right)=0, \\
& -v_{g} \frac{\partial v_{z}}{\partial \xi}+v_{x} \frac{\partial v_{z}}{\partial x}+v_{z} \frac{\partial v_{z}}{\partial \xi}-\frac{e}{m}\left(\frac{\partial \phi}{\partial \xi}+v_{y} B_{x 0} \sin \left(\frac{\pi x}{b}\right)\right)=0, \\
& \left(n_{0}+n_{e 1}\right) v_{y}+\frac{\sin (\pi x / b)}{\mu_{0} e}\left(\frac{\pi}{b} B_{z 0}+\frac{\partial B_{x 0}}{\partial \xi}+\frac{v_{g}}{c^{2}} \frac{\partial E}{\partial \xi}\right)=0, \\
& \frac{\partial E}{\partial \xi}+v_{g} \frac{\partial B_{x}}{\partial \xi}=0, \\
& \frac{\pi}{b} E-v_{g} \frac{\partial B_{z}}{\partial \xi}=0, \\
& \frac{\partial^{2} \phi}{\partial \xi^{2}}-\frac{e}{\varepsilon_{0}} n_{e 1}=0 .
\end{aligned}
$$

Here $v_{g}=c \sqrt{\left(1-\omega_{p}^{2} / \omega^{2}-\pi^{2} c^{2} / b^{2} \omega^{2}\right)}$ is the group velocity of the microwave. Now we integrate Eq. (7) under the condition that all the oscillating quantities vanish far away, i.e. as $\xi \rightarrow \infty$ in order to obtain

$$
\left(v_{z}-v_{g}\right)\left(n_{0}+n_{e 1}\right)=-n_{0} v_{g} .
$$

From Eq. (11) and Eq. (15) we calculate $v_{y}$ and $v_{z}$, respectively. Their substitution in Eq. (10) leads

$$
\begin{aligned}
& \frac{n_{0} v_{g}^{2}}{n_{0}+n_{e 1}} \frac{\partial}{\partial \xi}\left(\frac{n_{e 1}}{n_{0}+n_{e 1}}\right)+ \\
& \frac{e}{m} \frac{\partial \phi}{\partial \xi}-\frac{\sin ^{2}(\pi x / b)}{\mu_{0} m\left(n_{0}+n_{e 1}\right)} \\
& B_{x 0}\left(\frac{\pi}{b} B_{z 0}+\frac{\partial B_{x 0}}{\partial \xi}+\frac{v_{g}}{c^{2}} \frac{\partial E}{\partial \xi}\right)=0
\end{aligned}
$$

Now we simplify the above equation along with the use of weakly nonlinear theory which employs $n_{e 1} \ll n_{0}$. Therefore, we get

$$
\begin{aligned}
& v_{g}^{2} \frac{\partial n_{e 1}}{\partial \xi}+\left(n_{0}+n_{e 1}\right) \frac{e}{m} \frac{\partial \phi}{\partial \xi}-\frac{\sin ^{2}(\pi x / b)}{\mu_{0} m} \\
& B_{x 0}\left(\frac{\pi}{b} B_{z 0}+\frac{\partial B_{x 0}}{\partial \xi}+\frac{v_{g}}{c^{2}} \frac{\partial E}{\partial \xi}\right)=0
\end{aligned} .
$$

We put the value of $\partial n_{e 1} / \partial \xi$ from Eq. (14) in Eq. (17) and then integrate the resultant equation for obtaining

$$
\begin{gathered}
\phi^{\prime \prime}+\frac{e}{2 m v_{g}^{2}} \phi^{\prime 2}+\frac{\omega_{p}^{2}}{v_{g}^{2}} \phi-\frac{e c^{2}}{m v_{g}^{2}} \sin ^{2}\left(\frac{\pi x}{b}\right) \\
\int B_{x 0}\left(\frac{\pi}{b} B_{z 0}+\frac{\partial B_{x 0}}{\partial \xi}+\frac{v_{g}}{c^{2}} \frac{\partial E}{\partial \xi}\right) d \xi=0 .
\end{gathered}
$$

Here prime denotes the differentiation with respect to $\xi$. Using Eqs. (12) and (13) we eliminate $B_{x 0}$ and $B_{z 0}$ for Gaussian-like field profile of microwave pulse, given by $E(\xi)=E_{0}[1+\cos \{2 \pi(\xi-L / 2) / L\}]$ and shown in Fig. (2). Finally we achieve the main equation in terms of wake potential $\phi$ as follows

$$
\begin{gathered}
\phi^{\prime \prime}+\frac{e}{2 m v_{g}{ }^{2}} \phi^{\prime 2}+\frac{\omega_{p}{ }^{2}}{v_{g}^{2}} \phi-\frac{1}{2}\left(\frac{E_{0}{ }^{2} c^{2} e}{m v_{g}{ }^{4}}\right) \sin ^{2}\left(\frac{\pi x}{b}\right) \\
\times\left[\begin{array}{l}
\left(1-\frac{v_{g}{ }^{2}}{c^{2}}\right)[1+\cos \{2 \pi(\xi-L / 2) / L\}]^{2}- \\
\frac{\pi^{2}}{b^{2}}\left(\xi+\frac{L}{2 \pi} \sin \{2 \pi(\xi-L / 2) / L\}\right)^{2}
\end{array}\right]=0 .
\end{gathered}
$$

In order to analyze the physics behind the various terms of Eq. (19), we mention that this equation can be viewed as the equation governing the forced (driven) harmonic oscillator. In this equation, the last term is the force term that evolves due to the microwave field (ponderomotive force) and drives the wake in the plasma. The third term is proportional to $\phi$ and hence its coefficient determines the natural frequency of the wake. The second term is the damping term through which the nonlinearity enters the system as it is proportional to square of $\partial \phi / \partial \xi$ (nonlinear term). Finally we can say that the wake with potential $\phi$ is evolved in the plasma as a combined contribution of each term of Eq. (19). 


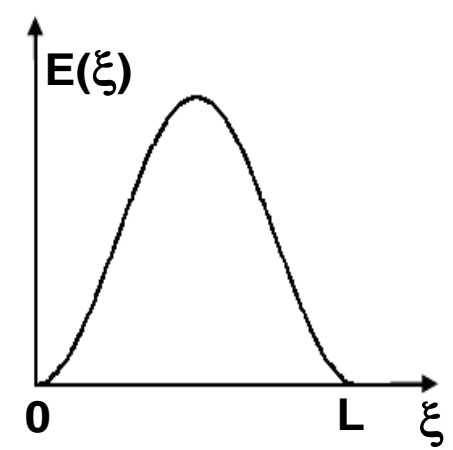

Fig. (2). Gaussian-like field profile of microwave pulse corresponding to $E(\xi)=E_{0}[1+\cos \{2 \pi(\xi-L / 2) / L\}]$.

\section{NUMERICAL STUDIES}

We solve Eq. (19) numerically for $\phi$ using fourth-order Runge-Kutta method by giving typical values to microwave intensity $I$ (corresponding field $E_{0}$ ), its frequency $f(=\omega / 2 \pi)$, equilibrium plasma density $n_{0}$ and waveguide width $b$. Here we consider the initial conditions as $\phi$ and its derivative $\partial \phi / \partial \xi$ to be zero at $\xi=L$ (front of the pulse). The microwave field is made confined within $\xi>0$ and $\xi<L$, i.e. $0 \leq \xi \leq L$. The boundary condition that the tangential component of electric field vanishes at the waveguide walls has already been used in obtaining the main governing equation (19). From the numerical results, we evaluate the wake behind the microwave pulse, i.e. at $\xi<0$. Then from the profile of wake potential, we find the associated wakefield which is used for the purpose of the particle acceleration.

Fig. (3) shows that the profile of the wake potential behind the microwave pulse when microwave intensity $I=1 \times 10^{9} \mathrm{~W} / \mathrm{m}^{2}$, microwave frequency $f=8 \mathrm{GHz}$, waveguide width $b=0.03 \mathrm{~m}$ and pulse duration $\tau=0.8 \mathrm{~ns}$ (corresponding plasma density $n_{0}=1.8 \times 10^{16} / \mathrm{m}^{3}$ ). Here it is worth noting that the wake potential of strength $1.6 \times 10^{5} \mathrm{~V}$ and the corresponding wakefield of about 3 $\mathrm{MV} / \mathrm{m}$ (Fig. 4) is obtained with the use of moderate intensity microwave pulse. In the present mechanism, it is expected that the wakefield of the order of $\mathrm{GV} / \mathrm{m}$ can be excited if the microwave pulse of intensity $\mathrm{TW} / \mathrm{m}^{2}$ are available, though in usual wakefield generation schemes ultrahigh intensity $\left(\sim 10^{20}\right.$ $\mathrm{V} / \mathrm{m}^{2}$ ) laser pulses are required $[6-11,21]$. In order to see the effect of microwave frequency on the wakefield structure, we have prepared Fig. (5) at $f=10 \mathrm{GHz}$. By comparing this figure with Fig. (4) we realize that the wakefield gets reduced for the larger microwave frequency. This is consistent with the lower wake potential (Fig. 6) attained by the microwave with higher frequency. Finally this can be investigated from Figs. (3-6) that the wakefield produced in the rectangular waveguide (bounded system) is dispersive in nature contrary to the one in unbounded plasmas.

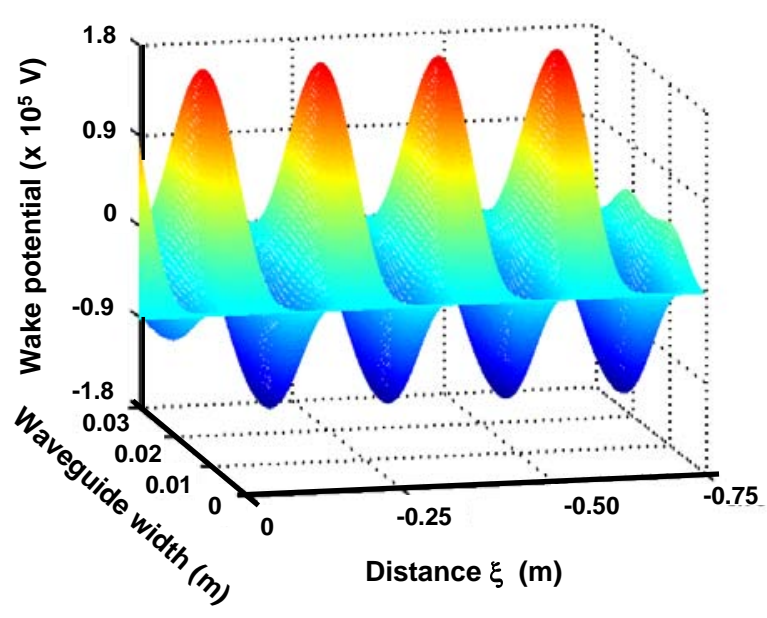

Fig. (3). Profile of wake potential when $I=1 \times 10^{9} \mathrm{~W} / \mathrm{m}^{2}, f=8 \mathrm{GHz}$, $b=0.03 m$ and $\tau=0.8 n s$ (corresponding plasma density $\left.n_{0}=1.8 \times 10^{16} / \mathrm{m}^{3}\right)$.

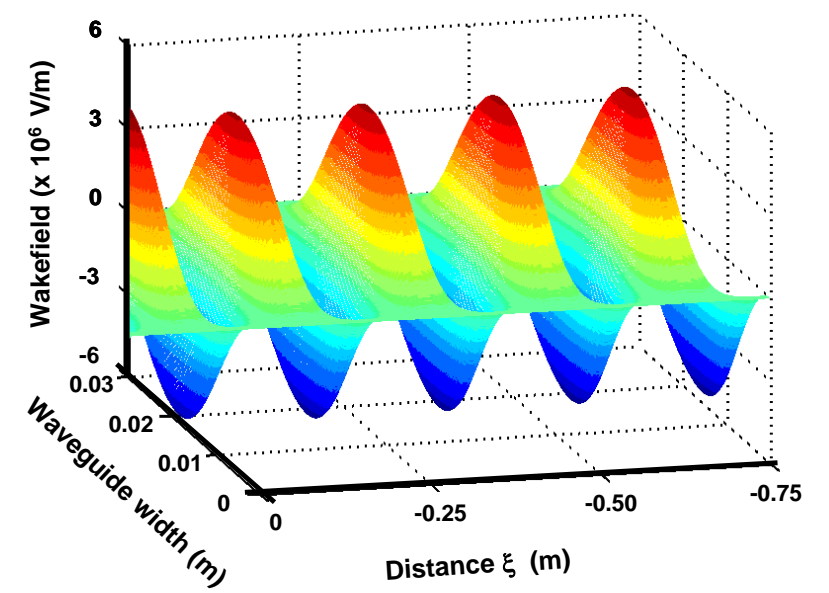

Fig. (4). Profile of wakefield $E_{W}$ corresponding to wake potential shown in Fig. (3).

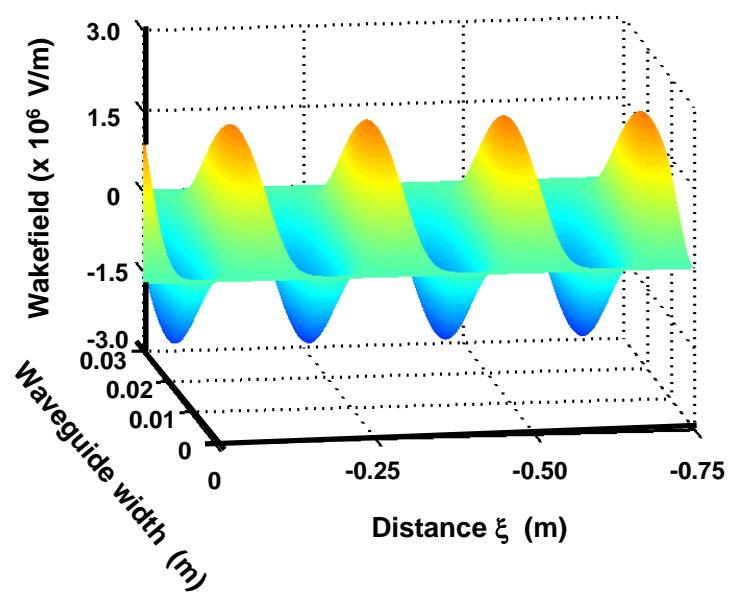

Fig. (5). Profile of wakefield for $f=10 \mathrm{GHz}$ when other parameters are the same as in Fig. (3). 


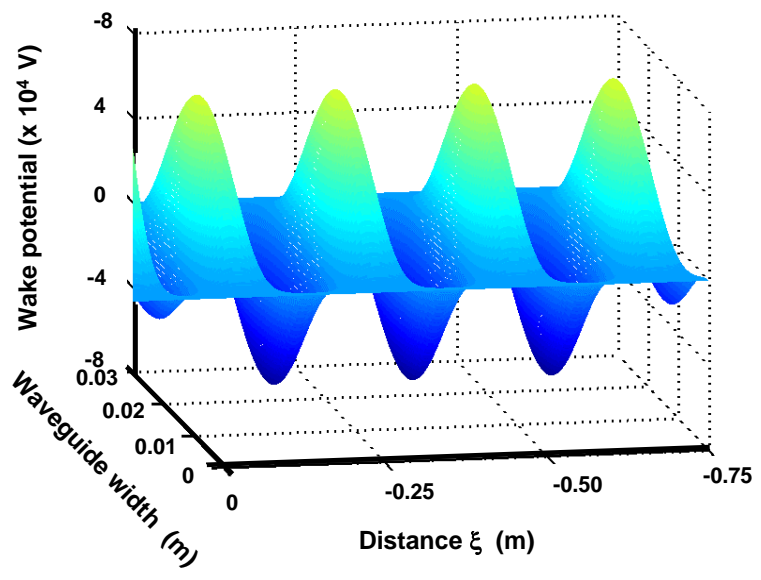

Fig. (6). Wake potential corresponding to Fig. (5), showing the effect of microwave frequency when compared with Fig. (3).

Figs. (7-10) show the effect of waveguide width $b$ and the microwave pulse duration $\tau$ on the structure of wake potential and the wake field. Here it is evident that the wake with lower potential is developed in the waveguide of larger width (compare Figs. (7) and (3)). The lower wake potential corresponds to the smaller wakefield (Fig. 8). However, it is evident from Fig. (9) that the larger amplitude wakefield is excited by a longer microwave pulse (compare Fig. (9) with Fig. (4)). Under this situation, wake with higher potential is correspondingly occurred (Fig. 10).

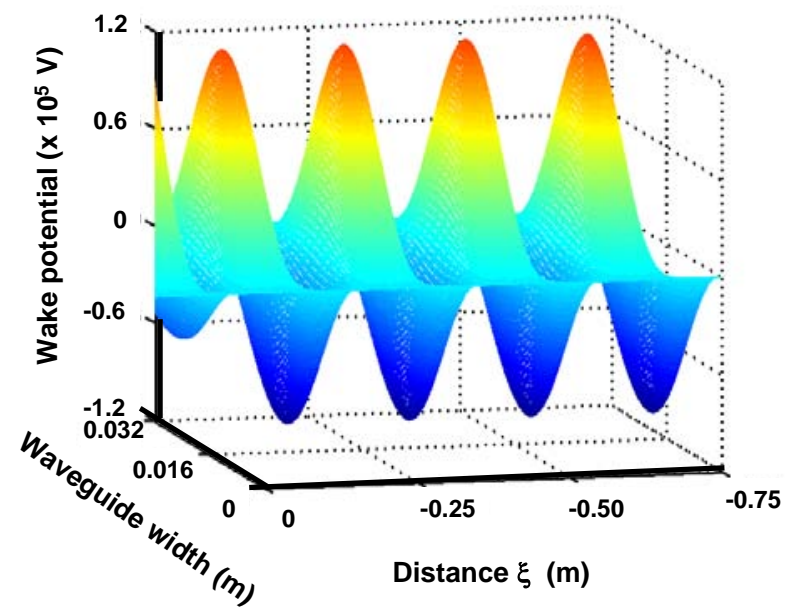

Fig. (7). Structure of wake potential achieved in larger width waveguide $(b=0.032 \mathrm{~m})$ for the other parameters the same as in Fig. (3).

Now we focus on the maximum amplitude of the wakefield $E_{W}$ under the effect of waveguide width and microwave parameters. The effects of microwave frequency $f$ and the intensity $I$ on this wakefield amplitude is shown in Fig. (11) for the same parameters as used in Fig. (3). It is evident here that the wakefield of lower strength is obtained at the higher frequency of microwave. The decreased wakefield for larger microwave frequency can be explained on the

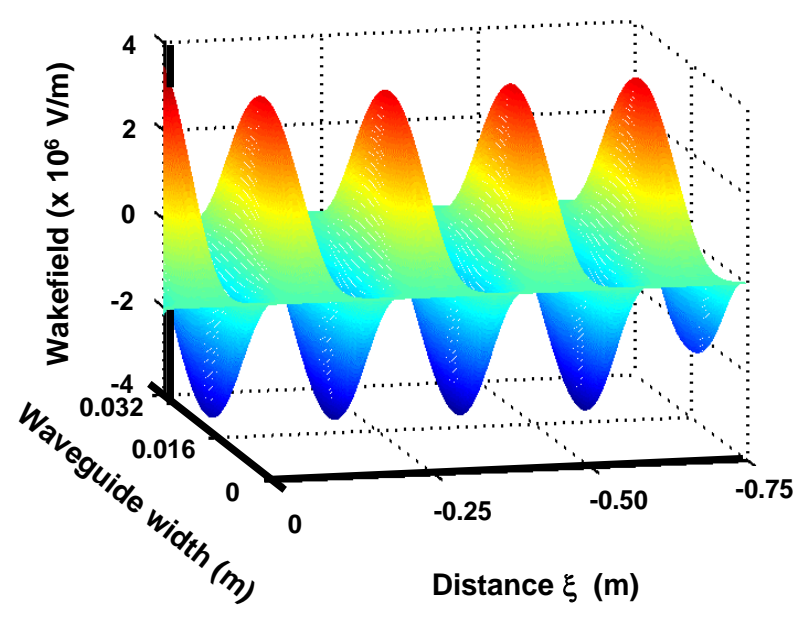

Fig. (8). Wakefield profile in the waveguide corresponding to Fig. (7) for the larger waveguide width.

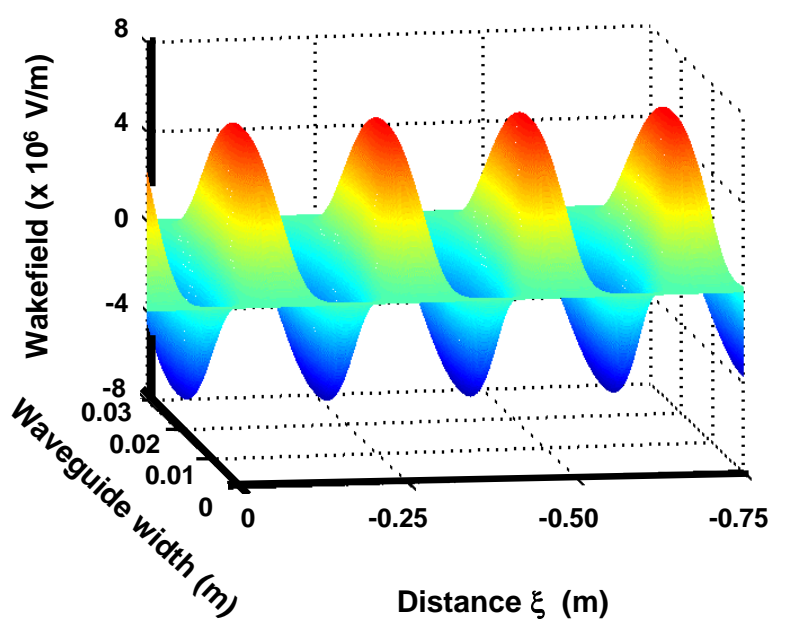

Fig. (9). Wakefield achieved by longer microwave pulse ( $\tau=0.9 n s$ ) for other parameters the same as in Fig. (3).

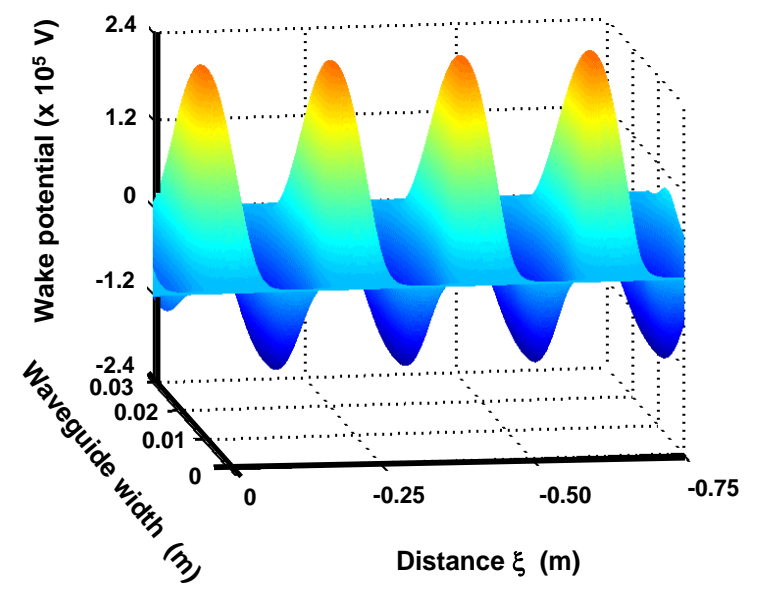

Fig. (10). Profile of wake potential for $\tau=0.9 n s$ and when other parameters are the same as in Fig. (3). 


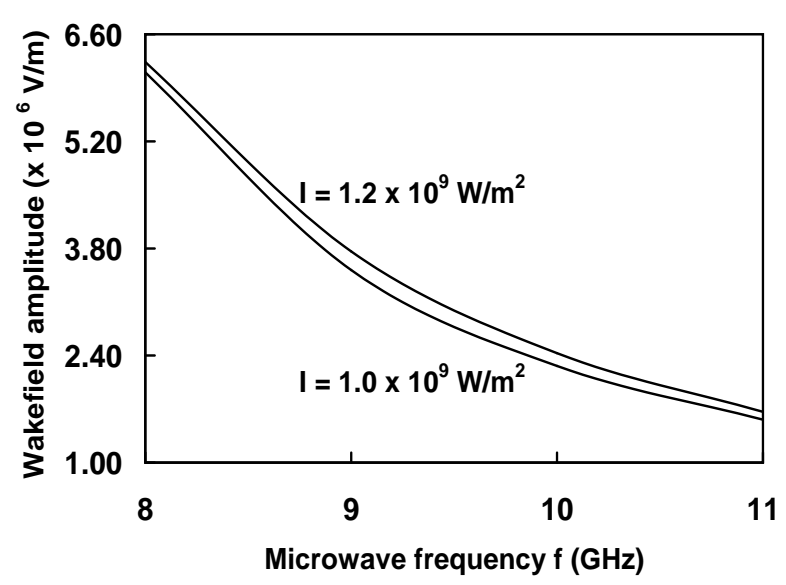

Fig. (11). Variation of maximum amplitude of the wakefield with microwave frequency for two different values of microwave intensity when other parameters are the same as in Fig. (3).

basis of force term in Eq. (19). Actually the group velocity $v_{g}$ gets larger with the increasing frequency $f$. This leads to weaker driven force via last term in Eq. (19). Therefore, the wake with smaller amplitude is excited in the waveguide. Also, the wakefield is found to be sensitive to the microwave frequency and it gets reduced from $6 \mathrm{MV} / \mathrm{m}$ to $1.7 \mathrm{MV} / \mathrm{m}$ when the frequency is increased from $8 \mathrm{GHz}$ to $11 \mathrm{GHz}$. However, the wakefield amplitude gets increased for the higher microwave intensity. Similar effects of the microwave frequency and intensity on wakefield amplitude have been observed in the case of laser induced wakefield in an unbounded plasma, where analytical calculations were carried out [6].

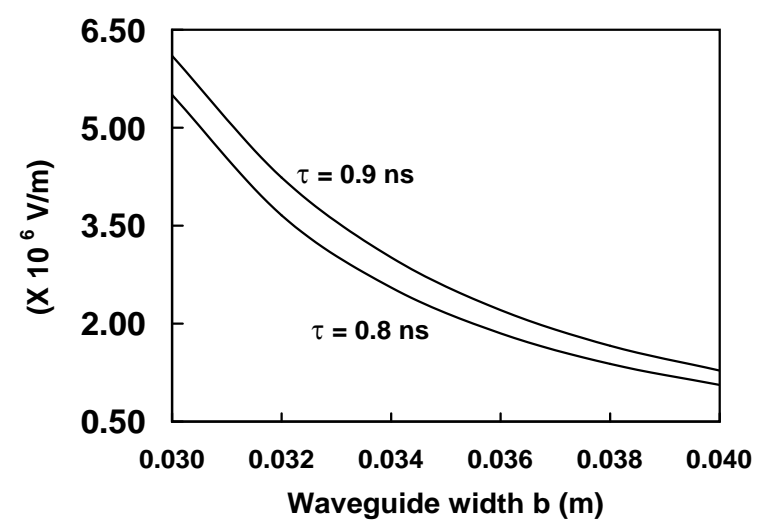

Fig. (12). Dependence of maximum amplitude of wakefield $E_{W}$ on waveguide width $b$ for two different values of microwave pulse duration $\tau$ and when other parameters are the same as in Fig. (3).

In Fig. (12) we show the dependence of maximum amplitude of wakefield $E_{W}$ on the waveguide width $b$ and pulse duration $\tau$ when $I=1 \mathrm{GW} / \mathrm{m}^{2}$ and $f=8 \mathrm{GHz}$. Here it is clear that larger amplitude wakefield is achieved when longer microwave pulse (large $\tau$ ) is used in the narrower waveguide (small $b$ ). The reason of the higher wakefield for the longer pulse duration can be understood on the basis of density perturbations. Actually for longer pulse durations the condition $\tau=f_{p}^{-1}$ is satisfied for lower density of the plasma in the waveguide. Since we are keeping the microwave intensity fixed, due to the lower density in the waveguide higher density perturbations would be achieved for larger pulse durations. Consequently, larger magnitude wakefield would be produced by the higher density perturbations. The opposite behaviour of wakefield amplitude with the waveguide width is also evident from Fig. (12) and the amplitude $E_{W}$ gets reduced when the wakefield is excited in a wider waveguide by the microwave pulse of same parameters. Here it would be worth mentioning that under the larger waveguide width the force term of Eq. (19) gets smaller due to the increased velocity $v_{g}$. Hence the smaller amplitude wakefield is achieved. Further, this can be seen that the wakefield is sensitive to the waveguide width.

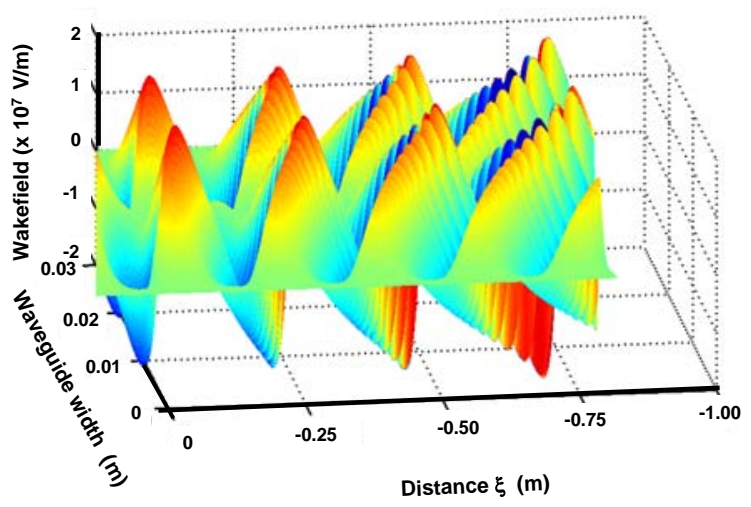

Fig. (13). Profile of wakefield $E_{W}$ in a plasma filled waveguide near cutoff conditions when $f=6.76 \mathrm{GHz}, \tau=0.9 \mathrm{~ns}$, $f_{c}=5.11 \mathrm{GHz}$ and all other parameters are the same as in Fig. (3).

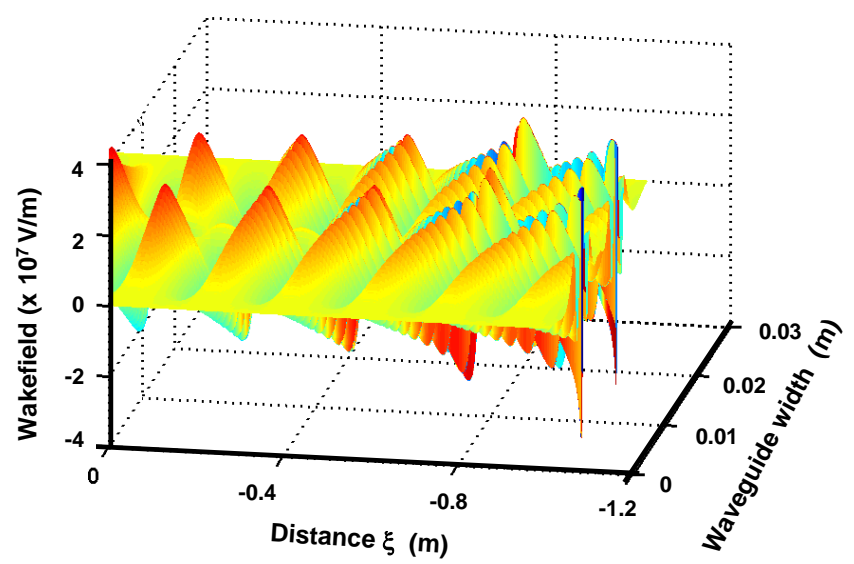

Fig. (14). Profile of wakefield corresponding to Fig. (13), showing its cutoff around maximum distance of $\xi=3.7 \mathrm{~L}$ from the microwave pulse.

\section{WAKEFIELD NEAR CUTOFF CONDITIONS}

From the above Figs. (3-12), we can observe that the wakefield of larger amplitude is obtained for the smaller waveguide width and the longer pulse duration. Since the 
cutoff frequency $f_{c}$ gets higher under the effect of decreased width $b$ and plasma density $n_{0}$, for fixed microwave frequency these effects can be viewed as if the microwave frequency is brought near the cutoff frequency $f_{c}$. Therefore, it would be of much interest to investigate the wakefield structure near cutoff conditions, i.e. when $f$ is near cutoff frequency $f_{c}$. We present these results in Figs. (13) and (14) for $f=6.76 \mathrm{GHz}$ whereas $f_{c}=5.11 \mathrm{GHz}$. It is clear from Fig. (13) that under this situation the amplitude of wake wave gets increased as we move away from the microwave pulse, i.e. for decreasing values of $\xi$. Further it can be noticed that various peaks develop along waveguide width during the growth of wakefield amplitude and it becomes unstable. Therefore, it appears that some instability develops near the cutoff conditions. In order to further investigate this effect, we show in Fig. (14) the maximum distance by which this growth occurs. Here, we observe that the amplitude gets terminated around $\xi=3.7 \mathrm{~L}$ in the plasma and the field breaks down.

In view of the above results, it is suggested that the microwave of higher frequency should be employed so that any instability does not grow in the system and the wakefield can be effectively used for the purpose of particle acceleration. The present mechanism of wakefield generation can be realized experimentally if we use wider waveguide filled with higher plasma density. The high density plasma can be produced in the waveguide under the action of Electron Cyclotron Resonance (ECR) action. However, under such situation short microwave pulses would be more effective in order to resonantly excite the plasma wake wave.

\section{CONCLUDING REMARKS}

Based on our numerical studies we observed that the effects of microwave frequency and waveguide width are to reduce the strength of wakefield. However, larger amplitude wakefield can be generated under the situation of longer pulse duration and higher intensity of microwave. In view of all the results, it is suggested that longer microwave pulses can excite appreciable wakefield in a narrow waveguide which is filled with lower density plasma. However, short microwave pulses would be effective if the microwave sources of higher frequency are available and a higher density plasma is used in the waveguide. In each case, higher intensity pulses produce higher amplitude wakefield due to the larger perturbations occurred in the plasma density. Further, the wakefield was found to be sensitive to the microwave frequency and waveguide width. Therefore, a judicious choice of microwave pulse parameters and the waveguide dimensions can lead to larger amplitude wake field for the effective particle acceleration.

\section{REFERENCES}

[1] Leemans WP, Rodgers D, Catravas P, et al. Gamma-neutron activation experiments using laser wakefield accelerators. Phys Plasmas 2001; 8: 2510-6.

[2] Santala MIK, Zepf M, Beg FN, et al. Production of radioactive nuclides by energetic protons generated from intense laser-plasma interactions. Appl Phys Lett 2001; 78: 19-21.
[3] Leemans WP, Geddes CGR, Faure J, et al. Observation of terahertz emission from a laser-plasma accelerated electron bunch crossing a plasma-vacuum boundary. Phys Rev Lett 2003; 91: 074802(1-4).

[4] Schroeder CB, Esarey E, Tilborg JV, Leemans WP. Theory of coherent transition radiation generated at a plasma-vacuum interface. Phys Rev E 2004; 69: 016501(1-12).

[5] Leemans WP, Tilborg JV, Faure J, et al. Terahertz radiation from laser accelerated electron bunches. Phys Plasmas 2004; 11: 2899906.

[6] Malik HK, Kumar S, Nishida Y. Electron acceleration by laser produced wake field: Pulse shape effect. Opt Comm 2007; 280: 417-23.

[7] Lotov KV. Laser wakefield acceleration in narrow plasma-filled channels. Laser Part Beams 2001; 19: 219-22.

[8] Reitsma AJW, Jaroszynski DA. Coupling of longitudinal and transverse motion of accelerated electrons in laser wakefield acceleration. Laser Part Beams 2004; 22: 407-13.

[9] Lifschitz AF, Faure J, Glinec Y, Malka V, Mora P. Proposed scheme for compact $\mathrm{GeV}$ laser plasma accelerator. Laser Part Beams 2006; 24: 255-59.

[10] Flippo K, Hegelich BM, Albright BJ, et al. Laser-driven ion accelerators: Spectral control monoenergetic ions and new acceleration mechanisms. Laser Part Beams 2007; 25: 3-8.

[11] Nickles PV, Ter-avetisyan S, Schnuerer M, et al. Review of ultrafast ion acceleration experiments in laser plasma at Max Born Institute. Laser Part Beams 2007; 25: 347-63.

[12] Leemans WP, Volfbeyn P, Guo KZ, et al. Laser-driven plasmabased accelerators: Wakefield excitation, channel guiding and laser triggered particle injection. Phys Plasmas 1998; 5: 1615-23.

[13] Andreev NE, Kuznetsov SV. Laser wakefield acceleration of short electron bunches. IEEE Trans Plasma Sci 2000; 28: 1170-6.

[14] Malka V, Fritzler S, Lefebvre E, et al. Electron acceleration by a wake field forced by an intense ultrashort laser pulse. Science 2002; 298: 1596-600.

[15] Gorbunov LM, Mora P, Solodov AA. Dynamics of a plasma channel created by the wakefield of a laser pulse. Phys Plasmas 2003; 10:1124-34.

[16] Shvets G, Fisch JN, Pukhov A. Excitation of accelerating plasma waves by counter-propagating laser beams. Phys Plasmas 2002; 9: 2383-92.

[17] Najmudin Z, Krushelnick K, Clark EL, et al. Self-modulated wakefield and forced laser wakefield acceleration of electrons. Phys Plasmas 2003; 10: 2071-7.

[18] Gorbunov LM, Mora P, Ramazashvili RR. Laser surface wakefield in a plasma column. Phys Plasmas 2003; 10: 4563-6.

[19] Balakirev BB, Karas VI, Karas IV, Levchenko VD. Plasma wakefield excitation by relativistic electron bunches and charged particle acceleration in the presence of external magnetic field. Laser Part Beams 2001; 19: 597-604.

[20] Zhou CT, Yu MY, He XT. Electron acceleration by high currentdensity relativistic electron bunch in plasmas. Laser Part Beams 2007; 25: 313-9.

[21] Kingham RJ, Bell AR. Enhanced wakefields for the 1D laser wakefield accelerator. Phys Rev Lett 1997; 79: 4810-3.

[22] Andreev NE, Chegotov MV, Veisman ME. Wakefield generation by elliptically polarized femtosecond laser pulse in ionizing gases. IEEE Trans Plasma Sci 2000; 28: 1098-105.

[23] Cros B, Courtois C, Malka G, et al. Extending plasma accelerators: guiding with capillary tubes. IEEE Trans Plasma Sci 2000; 28: 1071-7.

[24] Sprangle P, Hafizi B, Peñano JR, et al. Wakefield generation and $\mathrm{GeV}$ acceleration in tapered plasma channels. Phys Rev E 2001; 63: 056405(1-11).

[25] Kado M, Daido H, Fukumi A, et al. Observation of strongly collimated proton beam from Tantalum targets irradiated with circular polarized laser pulses. Laser Part Beams 2006; 24: 117-23.

[26] Karmakar A, Pukhov A. Collimated attosecond GeV electron bunches from ionization of high-Z material by radially polarized ultra-relativistic laser pulses. Laser Part Beams 2007; 25: 371-7.

[27] Xu JJ, Kong Q, Chen Z, et al. Polarization effect of fields on vacuum laser acceleration. Laser Part Beams 2007; 25: 253-7.

[28] Zhang TB, Hirshfield JL, Marshall TC, Hafizi B. Stimulated dielectric wake-field accelerator. Phys Rev E 1997; 56: 4647-55.

[29] Park SY, Hirshfield JL. Theory of wakefields in a dielectric-lined waveguide. Phys Rev E 1997; 62: 1266-83. 
[30] Jing C, Liu W, Xiao I, Gai W, Schoessow P. Dipole-mode wakefields in dielectric-loaded rectangular waveguide accelerating structures. Phys Rev E 2003; 68: 016502 (1-6).

[31] Nishida Y, Yoshizumi M, Sugihara R. Electron acceleration by electromagnetic waves in a weakly magnetized inhomogeneous plasma. Phys Fluids 1985; 28: 1574-6.

[32] Nishida Y, Sato N. Observation of high-energy electrons accelerated by electrostatic waves propagating obliquely to a magnetic field. Phys Rev Lett 1987; 59: 653-6.

[33] Nishida Y, Shinozaki T. Resonant wave-particle interactions in $\vec{v}_{p} \times \vec{B}$ acceleration scheme. Phys Rev Lett 1990; 65: 2386-9.

[34] Nishida Y, Okazaki T, Yugami N, Nagasawa T. Excitation of large-amplitude ion-wave wake fields. Phys Rev Lett 1991; 66: 2328-31.
[35] Nishida Y, Kusaka S, Yugami N. Excitation of wakefield and electron acceleration by short microwave pulse. Physica Scripta 1994; T52: 65-8.

[36] Jawla SK, Kumar S, Malik HK. Evaluation of mode fields in a magnetized plasma waveguide and electron acceleration. Opt Comm 2005; 251: 346-60.

[37] Kumar S, Malik HK. Electron acceleration in a plasma filled rectangular waveguide under obliquely applied magnetic field. J Plasma Phys 2006; 72: 983-7.

[38] Malik HK. Application of obliquely interfering $\mathrm{TE}_{10}$ modes for electron energy gain. Opt Comm 2007; 278: 387-94.

[39] Tajima T, Dawson JM. Laser electron accelerator. Phys Rev Lett 1979; 43: 267-270.

[40] Ting A, Esarey E, Sprangle P. Nonlinear wakefield generation and relativistic focusing of inense laser pulses in plasmas. Phys Fluids B 1990; 2: 1390-4.

(C) Aria and Malik; Licensee Bentham Open.

This is an open access article licensed under the terms of the Creative Commons Attribution Non-Commercial License (http://creativecommons.org/licenses/by$\mathrm{nc} / 3.0 /$ ) which permits unrestricted, non-commercial use, distribution and reproduction in any medium, provided the work is properly cited. 